\title{
Fecal Microbiota Transplantation beyond Clostridioides Difficile Infection
}

\author{
Chang Mo Moon ${ }^{1}$ and Sung Noh Hong ${ }^{2}$ \\ ${ }^{1}$ Department of Internal Medicine, Ewha Womans University College of Medicine, Seoul, ${ }^{2}$ Department of Medicine, Samsung Medical \\ Center, Sungkyunkwan University School of Medicine, Seoul, Korea
}

With advancing analytical methods for gut microbes, many studies have been conducted, revealing that gut microbes cause various diseases, including gastrointestinal and non-gastrointestinal diseases. Accordingly, studies have been actively conducted to analyze the effects on the prevention and treatment of these diseases through changes in intestinal microbes and control of dysbiosis. Fecal microbiota transplantation (FMT) is an effort and is currently being applied to Clostridioides difficile treatment in Korea. Many studies have demonstrated the application of FMT in inflammatory bowel disease, irritable bowel syndrome, non-alcoholic fatty liver disease, metabolic syndrome, obesity, and diabetes. With further studies and accumulation of evidence, FMT could help treat presently untreatable diseases in clinical practice. Clin Endosc 2021;54:149-151

Key Words: Dysbiosis; Fecal microbiota transplantation; Prevention; Treatment

\section{INTRODUCTION}

The number of microorganisms that coexist in the human body is 100 trillion, which is 100 times more than the number in human cells. Further, the number of genes in microorganisms is known to be over 100 times. ${ }^{1,2}$ The intestinal microbiome plays a physiological role in digestive function, including the digestion of polysaccharides in the human body, development and differentiation of the intestinal epithelium and immune system, maintenance of tissue homeostasis, resistance to infection, synthesis of vitamins, and balancing of intestinal microbes. ${ }^{3}$ Presently, functional and pathological changes occur because of alterations in composition, richness, and/or diversity of the intestinal microbiota, or "dysbiosis". Although changes in the composition and imbalance of intestinal mi-

Received: February 3, 2021 Revised: February 24, 2021

Accepted: February 25, 2021

Correspondence: Sung Noh Hong

Department of Medicine, Samsung Medical Center, Sungkyunkwan University School of Medicine, 81 Irwon-ro, Gangnam-gu, Seoul 06351, Korea

Tel: +82-2-3410-3409, Fax: +82-2-3410-6983, E-mail: gisnhong@gmail.com ORCID: https://orcid.org/0000-0002-4140-3717

It is the invited review article.

(c) This is an Open Access article distributed under the terms of the Creative Commons Attribution Non-Commercial License (http://creativecommons.org/ licenses/by-nc/3.0) which permits unrestricted non-commercial use, distribution, and reproduction in any medium, provided the original work is properly cited. croflora occur in various diseases, whether these changes are the cause or effect of the disease is unclear. ${ }^{4}$ Recently, various attempts, including fecal microbiota transplantation (FMT), have been made to prevent and treat various diseases by controlling intestinal microbial imbalance. ${ }^{5,6}$ The aim of the present study was to determine the current status and prospects of the treatment of intestinal diseases, except Clostridioides difficile infection and other diseases through FMT.

\section{INFLAMMATORY BOWEL DISEASE}

Isolation of microbes from the gastrointestinal tract of patients with inflammatory bowel disease (IBD) reveals that the diversity of microbiota is reduced compared to that in healthy people. This phenomenon is seen in both ulcerative colitis (UC) and Crohn's disease, which are two major IBDs. UC is associated with decreased Akkermansia muciniphila, and Crohn's disease is associated with decreased Faecalibacterium prausnitzii. ${ }^{7,8}$ In animal IBD models, intestinal microbes cause disease. Further, in Crohn's disease, if stool flow alters, inflammation of the lower intestine improves. Probiotics are effective in preventing pouchitis in UC, ${ }^{9}$ suggesting that the gut microbiota plays an important role in the development of IBD.

In a recent meta-analysis of four double-blind randomized 
controlled trials (RCTs) involving patients with UC, clinical remission was observed in $28 \%$ of cases in the stool transplant group and $9 \%$ of cases in the placebo group. These results suggest that stool transplantation has a moderate therapeutic effect in patients with active UC, but applying the results directly to clinical practice is difficult because of differences in methods across studies. ${ }^{10}$

The persistence of response to FMT in UC is unclear, with a few studies reporting long-term outcomes. ${ }^{11}$ Two RCTs evaluating remission induction in UC reported 1-year outcomes in patients with primary outcomes at week 8 . In a study by Costello et al., ${ }^{12}$ five of $12(42 \%)$ patients with steroid-free remission (clinical and endoscopic) after donor FMT showed maintenance of remission for 12 months. In a study by Moayyedi et al., eight of nine patients showed maintenance of clinical remission at 9-12 months, but some patients received FMT monthly. ${ }^{13}$ There is no consensus on FMT as a UC treatment, and a recent consensus addressed that FMT should be performed for UC in a clinical trial setting.

\section{IRRITABLE BOWEL SYNDROME}

The mechanism of irritable bowel syndrome (IBS) development is unclear. A hypothesis to be explained is intestinal dysbiosis. ${ }^{14,15}$ In animal experiments, intestinal bacteria play an important role in digestive tract motility. Germ-free mice show delayed gastric emptying time and intestinal pain, which is normalized by the administration of normal intestinal bacteria. ${ }^{16,17}$ The expressions of Esherichia, Shigella, and Aeromonas increase and those of Actinobacter, Citrobacter, and Microvirgula decrease in patients with IBS. ${ }^{18}$ After FMT in 45 patients with IBS with chronic constipation, $60 \%$ of patients recovered, and $60 \%$ returned to a normal state $9-19$ months later. ${ }^{19}$

A recent systematic review and meta-analysis analyzed five RCTs including 267 patients with IBS, in which $92 \%$ of patients had IBS with diarrhea or mixed bowel habits. ${ }^{20}$ FMT failed to improve the symptoms of IBS (relative risk [RR] , 0.98; 95\% confidence interval [CI], 0.58-1.66). FMT using donor stool via colonoscopy was superior in two RCTs (RR, 0.63; 95\% CI, 0.43-0.93) and via a nasojejunal tube in one RCT (RR, $0.69 ; 95 \% \mathrm{CI}, 0.46-1.02$ ) compared to those using autologous stool. Further large-scale RCTs are required to reach definitive conclusions.

\section{NON-ALCOHOLIC FATTY LIVER DISEASE}

The pathogenesis of non-alcoholic fatty liver disease (NAFLD) is unknown, but hepatic focal deposition, liver damage, and imbalance of intestinal bacteria may be involved. Studying microorganisms in the patient's feces using pyrosequencing or quantitative polymerase chain reaction confirmed that the Ruminococcaceae family or Bacteroidetes phylum increased in patients compared to healthy individuals. ${ }^{21-23} \mathrm{~A}$ randomized controlled blinding method is underway to determine the effect of FMT on regression. ${ }^{19}$ Further research is warranted to identify the effect of FMT on NAFLD.

\section{METABOLIC SYNDROME AND OBESITY}

Alterations in the composition and metabolic capacity of gut microbiota in obesity promote adiposity and influence metabolic processes in the peripheral organs, such as the brain, liver, adipose tissue, and muscle. ${ }^{24}$ Metabolic syndrome occurs in more than $50 \%$ of patients with obesity and is associated with chronic inflammation. Translocation of bacteria in the intestine has been suggested as the cause of this metabolic endotoxemia. ${ }^{25}$ Several studies have revealed that obesity is associated with changes in the gut microbiota. The first evidence reported identified that the microbiota of leptin-deficient ob/ ob mice increases Firmicutes and decreases Bacteroidetes. ${ }^{26}$ In addition, when wild-type mice are administered with a highfat diet, Firmicutes increases, and Bacteroidetes decreases. ${ }^{27}$ With respect to obesity, the ratio of Firmicutes/Bacteroidetes increased in the gut microbiota of patients with obesity, and fraction of Bacteroidetes increased when a low-carb or low-fat diet was fed. ${ }^{28}$

When FMT was performed on 18 patients with metabolic syndrome using their own or the underweighted person's stool, nine patients who received stool from people with underweight body mass index (BMI) had noticeably reduced triglyceride levels and increased insulin sensitivity compared to the control group. ${ }^{29}$ In another study, 6 weeks after infusing microbiota from people with underweight BMI, the recipient's insulin sensitivity increased along with the level of intestinal microbiota producing butyrate. ${ }^{30}$

\section{DIABETES}

Regarding the role of intestinal microbes in the etiology of type 2 diabetes characterized by insulin resistance, administering a high-fat diet to mice increases intestinal permeability, resulting in elevated lipopolysaccharide levels, an outer membrane component of microbes, in the blood. Plasma lipopolysaccharide concentration could set the tone for insulin sensitivity and onsets of diabetes and obesity. ${ }^{31}$ Lactobacillus and Bifidobacterium were increased in diabetes-resistant mice, 
and Clostridiacea and Ruminococcacea were increased in diabetic-prone mice. ${ }^{32}$ In a randomized controlled, double-blind study including 18 men with insulin resistance who had not received previous treatment, 6 weeks after FMT, the insulin sensitivity and level of butyrate-producing intestinal microbes of recipients increased. ${ }^{30}$

\section{CONCLUSIONS}

Gut microbes are related to various diseases, with the breakthrough development of analytical methods. Inducing changes in gut microbes through FMT or probiotics has been actively researched to prevent and treat various diseases. Together with these studies, research is warranted on the method of safely performing FMT or best target diseases, and evidence needs to be obtained for use in clinical practice.

\section{Conflicts of Interest}

The authors have no potential conflicts of interest.

Funding
None.

ORCID

Chang Mo Moon:

https://orcid.org/0000-0003-2550-913X

Sung Noh Hong:

\section{REFERECES}

1. Ley RE, Peterson DA, Gordon JI. Ecological and evolutionary forces shaping microbial diversity in the human intestine. Cell 2006;124:837848.

2. Sekirov I, Russell SL, Antunes LCM, Finlay BB. Gut microbiota in health and disease. Physiol Rev 2010;90:859-904.

3. Belkaid Y, Hand TW. Role of the microbiota in immunity and inflammation. Cell 2014;157:121-141.

4. Chang C, Lin H. Dysbiosis in gastrointestinal disorders. Best Pract Res Clin Gastroenterol 2016;30:3-15.

5. Kim H, Kang K, Kim S, Im E. Recent undate in fecal microbiota transplantation. Korean Journal of Microbiology 2014;50:265-274.

6. Kang HY. Diseases and other factors affecting gut microbiota. In: 2017 Spring seminar of gastroenterology; 2017 April 16; Seoul, Korea. Seoul: Korean Society of Gastroenterology; 2017. p. 133-142.

7. Sokol H, Pigneur B, Watterlot L, et al. Faecalibacterium prausnitzii is an anti-inflammatory commensal bacterium identified by gut microbiota analysis of Crohn disease patients. Proc Natl Acad Sci U S A 2008;105:16731-16736.

8. Jostins L, Ripke S, Weersma RK, et al. Host-microbe interactions have shaped the genetic architecture of inflammatory bowel disease. Nature 2012;491:119-124.

9. Damman CJ, Miller SI, Surawicz, Zisman TL. The microbiome and inflammatory bowel disease: is there a therapeutic role for fecal microbiota transplantation? Am J Gastroenterol 2012;107:1452-1459.

10. Costello SP, Soo W, Bryant RV, Jairath V, Hart AL, Andrews JM. System-

atic review with meta-analysis: faecal microbiota transplantation for the induction of remission for active ulcerative colitis. Aliment Pharmacol Ther 2017;46:213-224.

11. Haifer C, Leong RW, Paramsothy S. The role of faecal microbiota transplantation in the treatment of inflammatory bowel disease. Curr Opin Pharmacol 2020;55:8-16.

12. Costello SP, Hughes PA, Waters O, et al. effect of fecal microbiota transplantation on 8-week remission in patients with ulcerative colitis: a randomized clinical trial. JAMA 2019;321:156-164.

13. Moayyedi P, Surette MG, Kim PT, et al. Fecal microbiota transplantation induces remission in patients with active ulcerative colitis in a randomized controlled trial. Gastroenterology 2015;149:102-109.e6.

14. Choung RS, Locke GR. Epidemiology of IBS. Gastroenterol Clin North Am 2011;40:1-10.

15. Mayer EA, Savidge T, Shulman RJ. Brain-gut microbiome interactions and functional bowel disorders. Gastroenterology 2014;146:1500-1512.

16. Abrams GD, Bishop JE. Effect of the normal microbial flora on gastrointestinal motility. Proc Soc Exp Biol Med 1967;126:301-304.

17. Husebye E, Hellström PM, Sundler F, Chen J, Midtvedt T. Influence of microbial species on small intestinal myoelectric activity and transit in germ-free rats. Am J Physiol Gastrointest Liver Physiol 2001;280:G368-G380.

18. Giamarellos-Bourboulis E, Tang J, Pyleris E, et al. Molecular assessment of differences in the duodenal microbiome in subjects with irritable bowel syndrome. Scand J Gastroenterol 2015;50:1076-1087.

19. Vrieze A, de Groot PF, Kootte RS, Knaapen M, van Nood E, Nieuwdorp M. Fecal transplant: a safe and sustainable clinical therapy for restoring intestinal microbial balance in human disease? Best Pract Res Clin Gastroenterol 2013;27:127-137.

20. Ianiro G, Eusebi LH, Black CJ, Gasbarrini A, Cammarota G, Ford AC. Systematic review with meta-analysis: efficacy of faecal microbiota transplantation for the treatment of irritable bowel syndrome. Aliment Pharmacol Ther 2019;50:240-248.

21. Zhu L, Baker SS, Gill C, et al. Characterization of gut microbiomes in nonalcoholic steatohepatitis (NASH) patients: a connection between endogenous alcohol and NASH. Hepatology 2013;57:601-609.

22. Mouzaki M, Comelli EM, Arendt BM, et al. Intestinal microbiota in patients with nonalcoholic fatty liver disease. Hepatology 2013;58:120-127.

23. Raman M, Ahmed I, Gillevet PM, et al. Fecal microbiome and volatile organic compound metabolome in obese humans with nonalcoholic fatty liver disease. Clin Gastroenterol Hepatol 2013;11:868-875.e1-e3.

24. Tremaroli V, Bäckhed F. Functional interactions between the gut microbiota and host metabolism. Nature 2012;489:242-249.

25. Nieuwdorp M, Gilijamse PW, Pai N, Kaplan LM. Role of the microbiome in energy regulation and metabolism. Gastroenterology 2014;146:1525-1533.

26. Ley RE, Bäckhed F, Turnbaugh P, Lozupone CA, Knight RD, Gordon JI. Obesity alters gut microbial ecology. Proc Natl Acad Sci U S A 2005;102:11070-11075.

27. Turnbaugh PJ, Bäckhed F, Fulton L, Gordon JI. Diet-induced obesity is linked to marked but reversible alterations in the mouse distal gut microbiome. Cell Host Microbe 2008;3:213-223.

28. Ley RE, Turnbaugh PJ, Klein S, Gordon JI. Microbial ecology: human gut microbes associated with obesity. Nature 2006;444:1022-1023.

29. Aroniadis OC, Brandt LJ. Fecal microbiota transplantation: past, present and future. Curr Opin Gastroenterol 2013;29:79-84.

30. Vrieze A, Van Nood E, Holleman F, et al. Transfer of intestinal microbiota from lean donors increases insulin sensitivity in individuals with metabolic syndrome. Gastroenterology 2012;143:913-916.e7.

31. Cani PD, Amar J, Iglesias MA, et al. Metabolic endotoxemia initiates obesity and insulin resistance. Diabetes 2007;56:1761-1772.

32. Roesch LF, Lorca GL, Casella G, et al. Culture-independent identification of gut bacteria correlated with the onset of diabetes in a rat model. ISME J 2009;3:536-548. 